\title{
Wind Forecasting Based on the HARMONIE Model and Adaptive Finite Elements
}

\author{
Albert Oliver ${ }^{1}$, Eduardo Rodríguez ${ }^{1}$, José María Escobar ${ }^{1}$, \\ Gustavo Montero ${ }^{1}$, Mariano Hortal ${ }^{2}$, Javier Calvo ${ }^{2}$, \\ José Manuel Cascón ${ }^{3}$, and Rafael Montenegro ${ }^{1}$ \\ ${ }^{1}$ University Institute for Intelligent Systems and Numerical \\ Applications in Engineering (SIANI), University of Las Palmas de \\ Gran Canaria (ULPGC) Campus de Tafira, 35017 Las Palmas de Gran \\ Canaria, Spain http://www.dca.iusiani.ulpgc.es/proyecto2012-2014 \\ ${ }^{2}$ Agencia Estatal de Meteorología (AEMET), Leonardo Prieto Castro, \\ 8, 28040 Madrid, Spain \\ ${ }^{3}$ Department of Economics and Economic History, Faculty of \\ Economics and Business, University of Salamanca, 37007 Salamanca, \\ Spain
}

\begin{abstract}
In this paper we introduce a new methodology for wind field forecasting over complex terrain. The main idea is to use the predictions of the HARMONIE mesoscale model as the input data for an adaptive finite element mass-consistent wind model. The HARMONIE results (obtained with a maximum resolution of about $1 \mathrm{~km}$ ) are refined in a local scale (about a few metres). An interface between both models is implemented in such a way that the initial wind field is obtained by a suitable interpolation of the HARMONIE results. Genetic algorithms are used to calibrate some parameters of the local wind field model in accordance to the HARMONIE data. In addition, measured data are considered to improve the reliability of the simulations. An automatic tetrahedral mesh generator, based on the meccano method, is applied to adapt the discretization to complex terrains. The main characteristic of the framework is a minimal user intervention. The final goal is to validate our model in several realistic applications in Gran Canaria island, Spain, with some experimental data obtained by the AEMET in their meteorological stations. The source code of the mass-consistent wind model is available on-line at http://www.dca.iusiani.ulpgc.es/Wind3D/
\end{abstract}




\section{Introduction}

Over the last years the use of wind power to produce electric power has augmented considerably. Wind models are tools that allow the study of several problems related to the atmosphere, such as, the effect of wind on structures, pollutant transport Oliver et al. [2012, 2013], fire spreading Ferragut et al. [2007], wind farm location Rodríguez [2004], etc.

In this paper we propose a methodology for wind forecasting by coupling the HARMONIE meso-scale model with a local mass-consistent wind model specially suited for complex terrain Rodríguez et al. [2012]; similar coupling methods have been proposed by Gasset et al. [2012] and Carvalho et al. [2013]. HARMONIE is used experimentally at AEMET with promising results Navascués et al. [2013]. Despite the high-resolution of the HARMONIE meso-scale model, the minimum horizontal resolution is about $1 \mathrm{~km}$, which is a drawback when the micro-scale (about $1 \mathrm{~m}$ ) is considered. For this reason the results of the HARMONIE meso-scale model are coupled with the local wind field model. An initial wind field is required: it is obtained by a vertical extrapolation and a horizontal interpolation. The vertical extrapolation is based on a log-linear wind profile Lalas and Ratto [1996]. Both the mass-consistent model and the interpolation are defined by a set of parameters. Some of these parameters are known, but others have to be estimated. In order to calibrate these parameters, genetic algorithms are used Montero et al. [2005]. Algorithm 1 synthesises the main steps of the model.

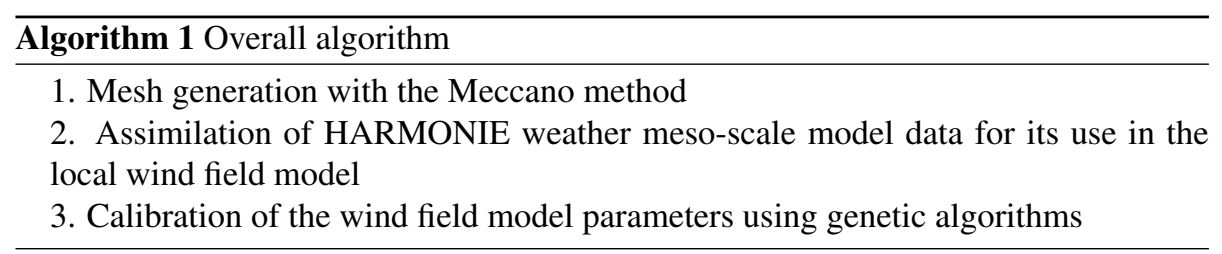

This paper is organised as follows. Sections 2, 3 and 4 explain in detail the main parts of this work: the meccano mesh generation (Section 2), the HARMONIE mesoscale model (Section 3) and the local wind field model (Section 4). Section 5 discusses the genetic algorithms and the parameters to be estimated. Section 6 shows an experiment of this method applied to Gran Canaria island.

\section{Meccano Mesh Generation}

The main steps of the meccano tetrahedral mesh generation algorithm are summarized in this section. This method has been previously introduced in Montenegro et al. [2009, 2010] and Cascón et al. [2013]. The input data of the algorithm is the definition of the solid boundary (for example a surface triangulation or CAD description) and a given precision (corresponding to the approximation of the solid boundary). Algorithm 2 describes our mesh generation approach.

The first step of the procedure is to construct a meccano approximation by connecting polyhedral pieces. The meccano and the solid must be equivalent from a topological point of view, i.e., their surfaces must have the same genus. 


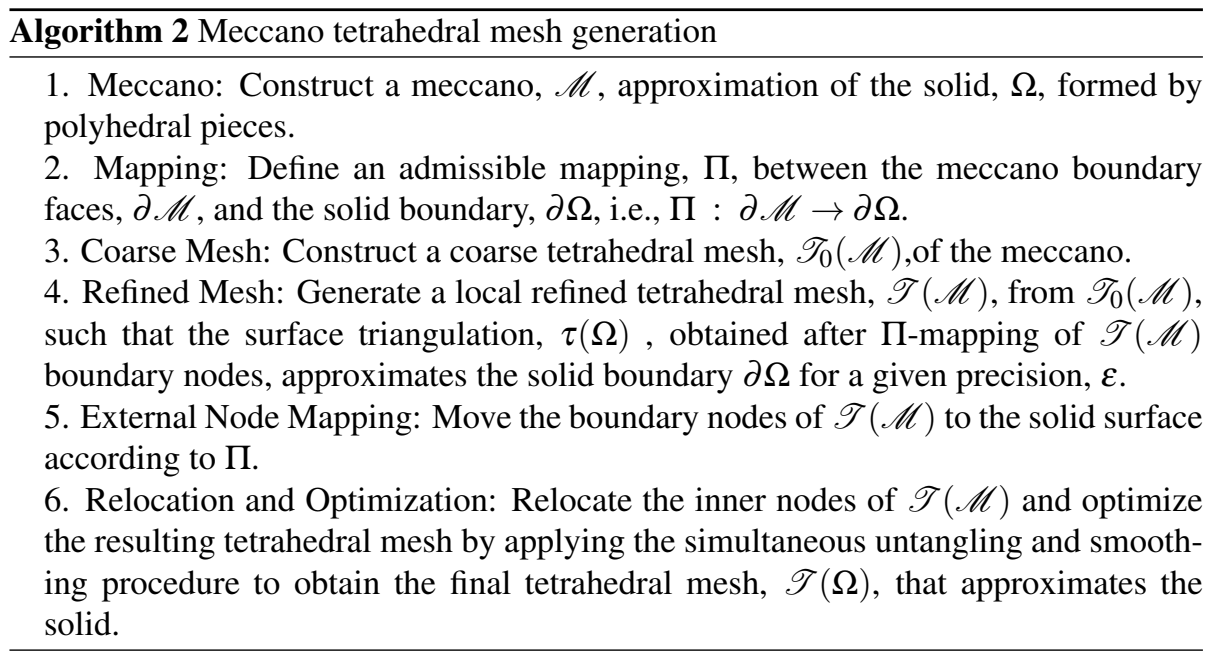

Figure 1: The different meccano steps

Once the meccano is assembled, we have to define an admissible one-to-one mapping between the boundary faces of the meccano and the boundary of the solid. If the solid is genus-zero and its boundary is given by a triangulation, we propose in Montenegro et al. [2010] an automatic method to construct a parametrization of the solid surface triangulation to a cube boundary. For this purpose, we first divide the solid surface triangulation into six patches with the same topological connection as the cube faces. Then, a discrete mapping from each surface patch to the corresponding cube face is built using the mean value parametrization proposed in Floater [2003].

At the moment, if the genus of the surface of the solid is greater than zero, the meccano should be defined by the user. An automatic construction of the meccano could be difficult when the topology of the solid is complex. We also remark that a non-optimal meccano can introduce large distortion in mesh generation. To avoid this issue an optimization of the boundary parametrization could be included Wan et al. [2011].

In step 3, the meccano is decomposed into a coarse tetrahedral mesh $\mathscr{T}_{0}(\mathscr{M})$ by an appropriate subdivision of its initial polyhedral pieces. Although any tetrahedralization algorithm could be used, we propose a partition of meccano compatible with the Kossaczký refinement algorithm Kossaczký [1994].

This mesh is locally refined in step 4 to obtain an approximation of the solid boundary within a given precision. To be more precise we have to introduce some notations. Given a tetrahedral mesh of the meccano $\mathscr{T}(\mathscr{M})$, we denote as $\tau(\mathscr{M})$ its boundary triangulation and $\tau(\Omega)$ the surface triangulation obtained after $\Pi$-mapping of $\tau(\mathscr{M})$ nodes. Note that $\tau_{o}(\Omega)$ is a coarse approximation of $\partial \Omega$. In order to improve this approximation we build a refined mesh $\mathscr{T}(\mathscr{M})$ of $\mathscr{T}_{0}(\mathscr{M})$ such that the distance between $\tau(\Omega)$ and $\partial \Omega$ is less than a prescribed tolerance $\varepsilon$. The concept of distance between surfaces can be defined and implemented in several ways. In our case it is as follows: 
Let $T=\langle a, b, c\rangle$ be a triangle of $\mathscr{T}(\mathscr{M})$, where $a, b$ and $c$ are their vertices, and let $p_{k} \in\left\{p_{i}\right\}_{i=1}^{N_{q}}$ be a Gauss quadrature point of $T$. We define the distance, $d(t)$, between the triangle $\langle\Pi(a), \Pi(b), \Pi(c)\rangle \in \tau(\Omega)$ and $\partial \Omega$ as the maximum of the volumes of the tetrahedra formed by $\Pi(a), \Pi(b), \Pi(c)$ and $\Pi\left(p_{k}\right)$. Then, the distance between $\tau(\Omega)$ and $\partial \Omega, d(\tau(\Omega)), \partial \Omega$, is the maximum of all $d(T)$, that is

$$
d(\tau(\Omega), \partial \Omega)=\max _{T \in \tau(\mathscr{M})} d(T)
$$

We recall that local refinement stops when $d(\tau(\Omega), \partial \Omega)<\varepsilon$. Note that this is an approximation of the maximum missed (or overestimated) volume per face of $\tau(\Omega)$. A more accurate approach of distance based on Hausdorff envelope can be found in Borouchaki and Frey [2005].

Then, we construct a mesh of the solid $\mathscr{T}(\Omega)$ by mapping the boundary nodes of $\mathscr{T}(\mathscr{M})$ and by relocating the inner nodes at a reasonable position. After these two steps, the resulting mesh is generally tangled. Therefore, a simultaneous untangling and smoothing procedure Escobar et al. [2003, 2010] is applied and a valid adaptive tetrahedral mesh of the solid is obtained. In short, this last procedure finds the new positions of the inner nodes of $\mathscr{T}(\Omega)$ optimizing an objective function. Such a function is based on a certain measurement of the quality of the local submesh $N(q)$, formed by the set of tetrahedra connected to the free node $q$. In fact, we use a suitable modification of the objective function such that it is regular over all $\mathbb{R}^{3}$, Escobar et al. [2003].

An example of the different steps of this method is shown in Fig. 1.

\section{HARMONIE Meso-scale Weather Model}

HARMONIE (HIRLAM-ALADIN Research on Meso-scale Operational NWP in Europe) is a weather prediction model design for operational use at convective scale resolutions. The system has been mainly developed by Meteo-France and ALADIN Consortium in collaboration with ECMWF and HIRLAM Consortium.

This model uses a 3D-Var data assimilation Fischer et al. [2005] which shares most of the code with the ECMWF and ARPEGE models. For surface variables a statistical interpolation algorithm is used. The Non-Hydrostatic Dynamics is based on Bubnová et al. [1995] and the physics is adapted from Meso-NH research model.

AEMET is running HARMONIE with AROME configuration at $2.5 \mathrm{~km}$ horizontal resolution since October 2011. This configuration is close to the one used operationally at Météo-France Seity et al. [2011]. Local and extreme forecasts are improved significantly with the HARMONIE $2.5 \mathrm{~km}$ model compared to coarser grid models like HIRLAM or ECMWF Navascués et al. [2013]. The model is run 4 times per day over 2 domains (Iberian Peninsula and Canary Islands) with a forecast length of 48 hours.

\section{Local Wind Field Simulation}

Once the tetrahedral mesh is constructed, we consider a mass-consistent model Montero et al. [1998, 2005], Ferragut et al. [2010] to compute a wind field $\mathbf{u}$ in the three- 
dimensional domain $\omega$, with a boundary $\Gamma=\Gamma_{a} \cup \Gamma_{b}$, that verifies the continuity equation and the impermeability condition on the terrain $\Gamma_{a}$,

$$
\begin{array}{ll}
\nabla \cdot \mathbf{u}=0 & \text { in } \omega \\
\mathbf{n} \cdot \mathbf{u}=0 & \text { on } \Gamma_{a}
\end{array}
$$

where $\mathbf{n}$ is the outward-pointing normal unit vector, being $\Gamma_{b}$ the boundary where the impermeability condition is not imposed.

The model formulates a least-squares problem in the domain $\omega$ to find a wind field $\mathbf{u}=(u, v, w)$, such that it is adjusted as much as possible to an interpolated wind field $\mathbf{u}_{0}=\left(u_{0}, v_{0}, w_{0}\right)$. The adjusting functional for a field $\mathbf{v}=(\widetilde{u}, \widetilde{v}, \widetilde{w})$ is defined as

$$
e(\mathbf{v})=\frac{1}{2} \int_{\omega}\left(\mathbf{v}-\mathbf{u}_{0}\right)^{t} \mathbf{p}\left(\mathbf{v}-\mathbf{u}_{0}\right) d \omega
$$

where $\mathbf{p}$ is a $3 \times 3$ diagonal matrix with $p_{1,1}=p_{2,2}=2 \alpha_{1}^{2}$ and $p_{3,3}=2 \alpha_{2}^{2}$. The Lagrange multiplier technique is used to minimise the functional (3), with the restrictions (2). Considering the Lagrange multiplier $\lambda$, the Lagrangian is defined as

$$
l(\mathbf{v}, \lambda)=e(\mathbf{v})+\int_{\omega} \lambda \nabla \cdot \mathbf{v} d \omega
$$

and the solution $\mathbf{u}$ is obtained by finding the saddle point $(\mathbf{u}, \phi)$ of the Lagrangian (4). This resulting wind field verifies the Euler-Lagrange equation,

$$
\mathbf{u}=\mathbf{u}_{\mathbf{0}}+\mathbf{p}^{-1} \nabla \phi
$$

where $\phi$ is the Lagrange multiplier. As $\alpha_{1}$ and $\alpha_{2}$ are constant in $\omega$, the variational approach results in an elliptic problem in $\phi$, by substituting (5) in (2), that is solved by using the finite element method.

$$
\begin{aligned}
-\nabla \cdot\left(\mathbf{p}^{-1} \nabla \phi\right) & =\nabla \cdot \mathbf{u}_{0} & & \text { in } \omega \\
-\mathbf{n} \cdot \mathbf{p}^{-1} \nabla \phi & =\mathbf{n} \cdot \mathbf{u}_{0} & & \text { on } \Gamma_{a} \\
\phi & =0 & & \text { on } \Gamma_{b}
\end{aligned}
$$

\subsection{Construction of the Initial Field}

The interpolated wind field $\mathbf{u}_{0}$ is constructed from the HARMONIE data, specifically the values of the $10 \mathrm{~m}$ wind, $\mathbf{u}_{n}^{h}$, given at point $n$ of the HARMONIE grid , the geostrophic wind $\mathbf{u}_{g}$. Therefore, we consider a horizontal interpolation and a vertical extrapolation of the available measurements to construct $\mathbf{u}_{0}$ in the whole computational domain.

\subsubsection{Horizontal Interpolation}

A common technique of interpolation at a given point, placed at a height $z_{m}$ over the terrain, is formulated as a function of the inverse of the squared distance between that 
point and the measurement stations, and the inverse of their height differences Montero et al. [1998]

$$
\mathbf{u}_{0}\left(z_{m}\right)=\xi \frac{\sum_{n=1}^{N} \frac{\mathbf{u}_{n}^{h}}{d_{n}^{2}}}{\sum_{n=1}^{N} \frac{1}{d_{n}^{2}}}+(1-\xi) \frac{\sum_{n=1}^{N} \frac{\mathbf{u}_{n}^{h}}{\left|\delta h_{n}\right|}}{\sum_{n=1}^{N} \frac{1}{\left|\delta h_{n}\right|}}
$$

where the value of $\mathbf{u}_{n}^{h}$ is the velocity measured at HARMONIE point $n, N$ is the number of HARMONIE points considered in the interpolation, $d_{n}$ is the horizontal distance from the station $n$ to the point of the domain where we are computing the wind velocity, $\left|\delta h_{n}\right|$ is the height difference between station $n$ and the studied point, and $\xi$ is a weighting parameter $(0 \leq \xi \leq 1)$, that allows to give more importance to one of these interpolation criteria.

\subsubsection{Vertical Extrapolation}

In this work, a log-linear wind profile is considered Lalas and Ratto [1996] in the surface layer, which takes into account the horizontal interpolation Montero and Sanín [2001] and the effect of roughness on the wind intensity and the direction. These values also depend on the air stability (neutral, stable or unstable atmosphere) according to the Pasquill stability class. Above the surface layer, a linear interpolation is carried out using the geostrophic wind. The logarithmic profile is given by,

$$
\mathbf{u}_{0}(z)=\frac{\mathbf{u}^{*}}{k}\left(\log \frac{z}{z_{0}}-\phi_{m}\right) \quad z_{0}<z \leq z_{s l}
$$

where $\mathbf{u}^{*}$ is the friction velocity, $k$ is von Karman's constant, $z_{0}$ is the roughness length McRae et al. [1982] and $z_{s l}$ is the height of the surface layer. The values of $\phi_{m}$ depend on the air stability. For neutral atmosphere its value is $\phi_{m}=0$, for stable atmosphere $\phi_{m}=-5 \frac{z}{l}$, and for unstable atmosphere

$$
\phi_{m}=\log \left[\left(\frac{\theta^{2}+1}{2}\right)\left(\frac{\theta+1}{2}\right)^{2}\right]-2 \arctan \theta+\frac{\pi}{2}
$$

where, $\theta=\left(1-16 \frac{z}{l}\right)^{1 / 4}$ and $\frac{1}{l}=a z_{0}^{b}$, with $a, b$, depending on the Pasquill stability class Zannetti [1990]. The friction velocity is obtained from (10) at any point $(x, y)$ by using the horizontal interpolated velocity $\mathbf{u}_{0}\left(z_{m}\right)$

$$
\mathbf{u}^{*}=\frac{k \mathbf{u}_{0}\left(z_{m}\right)}{\log \frac{z_{m}}{z_{0}}-\phi_{m}}
$$

The height of the boundary layer $z_{p b l}$ above the ground is chosen such that the wind intensity and direction are constant at that height,

$$
z_{p b l}=\frac{\gamma\left|\mathbf{u}^{*}\right|}{f}
$$


where $f=2 \omega \sin \phi$ is the Coriolis parameter ( $\omega$ is the Earth rotation and $\phi$ the latitude), and $\gamma$ is a parameter depending on the atmospheric stability, and being between 0.15 and 0.3 Panofsky and Dutton [1984]. The height of the mixed layer $h$ is considered to be equal to $z_{p b l}$ in neutral and unstable conditions. In stable conditions, it is approximated by

$$
h=\gamma \sqrt{\frac{\left|\mathbf{u}^{*}\right| l}{f}}
$$

where $\gamma^{\prime}=0.4$ Garratt [1982]. The height of surface layer is $z_{s l}=\frac{h}{10}$. From $z_{s l}$ to $z_{p b l}$, a linear interpolation with geostrophic wind $\mathbf{u}_{g}$ is carried out,

$$
\begin{aligned}
\mathbf{u}_{0}(z) & =\rho(z) \mathbf{u}_{0}\left(z_{s l}\right)+[1-\rho(z)] \mathbf{u}_{g} \quad z_{s l}<z \leq z_{p b l} \\
\rho(z) & =1-\left(\frac{z-z_{s l}}{z_{p b l}-z_{s l}}\right)^{2}\left(3-2 \frac{z-z_{s l}}{z_{p b l}-z_{s l}}\right)
\end{aligned}
$$

Finally, this model assumes $\mathbf{u}_{0}(z)=\mathbf{u}_{g}$ if $z>z_{p b l}$ and $\mathbf{u}_{0}(z)=0$ if $z \leq z_{0}$.

\section{Genetic Algorithms}

Genetic algorithms are optimisation tools based on the natural evolution mechanism. They produce successive trials that have an increasing probability to obtain a global optimum.

In this work we apply the model developed by Levine Levine [1994]. The most important aspects of genetic algorithms are the construction of an initial population, the evaluation of each individual in the fitness function, the selection of the parents of the next generation, the crossover of those parents to create the children, and the mutation to increase diversity. The initial population has been randomly generated and we use iteration limit exceeded as stopping criterion. The fitness function plays the role of the environment. It evaluates each string of a population. This is a measure, relative to the rest of the population, of how well that string satisfies a problem-specific metric. The values are mapped to a non-negative and monotonically increasing fitness value. Two population replacements are commonly used. The first, the generational replacement, replaces the entire population each generation Holland [1992]. The second, used in this work, is known as steady-state and only replaces a few individuals each generation Whitley [1989]. We have chosen the stochastic universal selection, and the uniform crossover operator Spears [1991]. The mutation operator is better used after crossover Davis [1991]. It allows to reach individuals in the search space that could not be evaluated otherwise. The mutation operator used in this work replaces the gene value with a random one within the initialisation range.

\subsection{Parameters to Calibrate}

In the numerical experiments with this wind model, the parameters to be estimated are $\alpha, \xi$ and $\gamma$. For this purpose, the fitting function to be minimised is the root mean 
square error (RMS) of the wind velocities given by the model with respect to the measures at the HARMONIE points.

$$
E(\alpha, \xi, \gamma)=\sqrt{\frac{\sum_{n=1}^{N}\left[\left(u_{n}^{\star}-u_{n}\right)^{2}+\left(v_{n}^{\star}-v_{n}\right)^{2}\right]}{2 N}}
$$

where $u_{n}^{\star}$ and $v_{n}^{\star}$ are the $X$ and $Y$ components of the HARMONIE wind field in the point $n$ used for the calibration, $u_{n}$ and $v_{n}$ are the $X$ and $Y$ components of the resulting wind field of the mass-consistent model (5), and $N$ is the number of points where the calibration is performed.

The first parameter to be estimated is the stability parameter $(\alpha)$, which is defined as

$$
\alpha=\frac{\alpha_{1}}{\alpha_{2}}
$$

where $\alpha_{1}$ and $\alpha_{2}$ are the components of the matrix $\mathbf{p}$ defined in (3). The parameter $\alpha$ defines the predominant component of the flow adjustment, being the vertical component when $\alpha>1$, and the horizontal component when $\alpha<1$.

The second parameter is the weighting coefficient $\xi$ in the equation (9). Note that $\xi=1$ implies the inverse of the squared distance interpolation, while $\xi=0$ stands for a height difference interpolation.

The parameter $\gamma$ is related to the height of the boundary layer $z_{p b l}$, and depends on the atmospheric stability. As stated in Section 4.1.2, its value varies in a range between 0.15 and 0.3 .

\section{Results}

In this section an example is presented using the methodology described in this paper. The example is located in Gran Canaria island, using the results from the HARMONIE model. Finally, a validation of the method is performed using measurement stations data.

\subsection{Mesh Generation}

The first step in the forecasting of the wind field is the generation of the air volumetric mesh. The mesh is created with the meccano method from a digital terrain model of the Gran Canaria island. The height of the domain is set to $10000 \mathrm{~m}$. The resulting mesh has 251808 nodes and 1090366 tetrahedra.

Figure 2 shows the terrain of the resulting meccano mesh and the terrain of the HARMONIE grid. The figure shows the difference in the discretization between the two models. This is the main motivation in coupling both models.

\subsection{HARMONIE Data for Mass-consistent Model}

The HARMONIE data assimilation has been done using the velocity at $10 \mathrm{~m}$, and the geostrophic wind. 


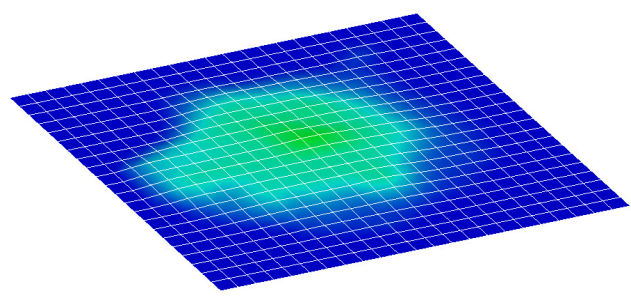

(a) HARMONIE grid

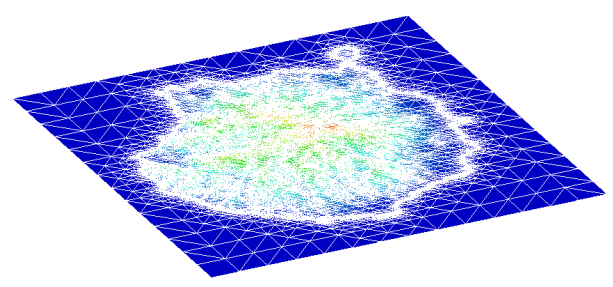

(b) Meccano mesh

Figure 2: Terrain discretization

The most straightforward method is to use the whole data at $10 \mathrm{~m}$, selecting a subset of that data as the measured data in the genetic process, and another subset as control points.

Figure 3 shows the terrain height in the meccano mesh and the HARMONIE grid. The great difference in heights indicates that probably not all values of the HARMONIE velocity at $10 \mathrm{~m}$ are appropriate. For this reason, we propose to use only those HARMONIE points whose height differ from the meccano height in less than a certain tolerance.

In Fig. 4, the points that will be used in the simulation are represented. The tolerance in this case has been set to $50 \mathrm{~m}$. As noted before, the points fulfilling the tolerance are divided in two different subsets, one used as stations (the green ones), and the other used as control points (the red ones).

\subsection{Model Calibration}

Once the stations and the control points are fixed, the calibration of the parameters can be done.

Using the genetic algorithm described above, a simulation of 30 genetic steps has been computed. Figure 5 shows the diminution of the fitting function (17) in the subsequent genetic steps.

The run time for an episode is about $90 \mathrm{~min}$ with a population size of 60 individuals using 6 cores in parallel. 


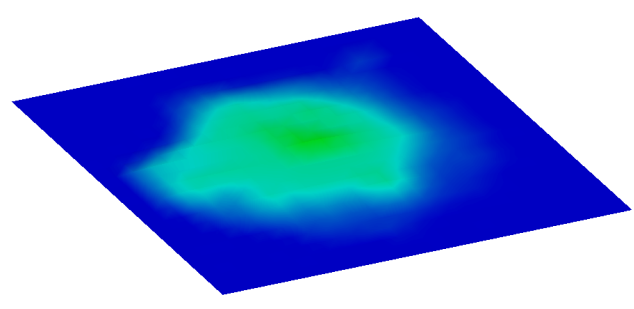

(a) HARMONIE grid

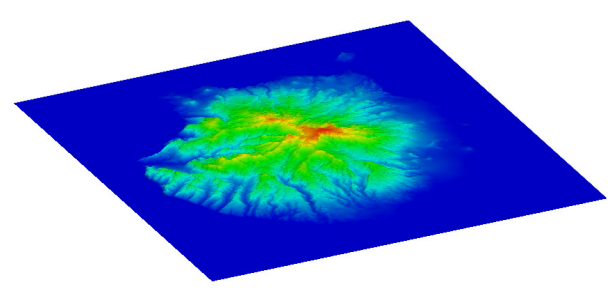

(b) Meccano mesh

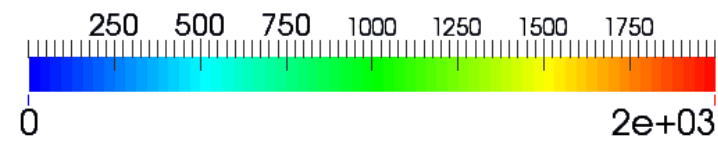

Figure 3: Terrain heights (m) 


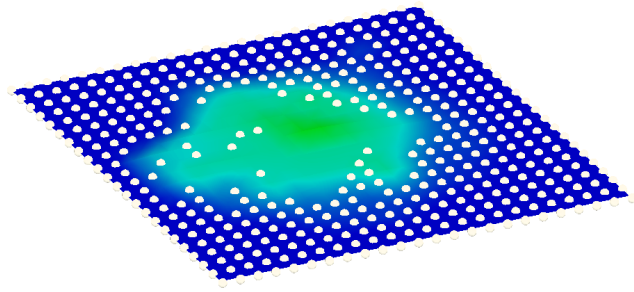

(a) Points fulfilling the tolerance

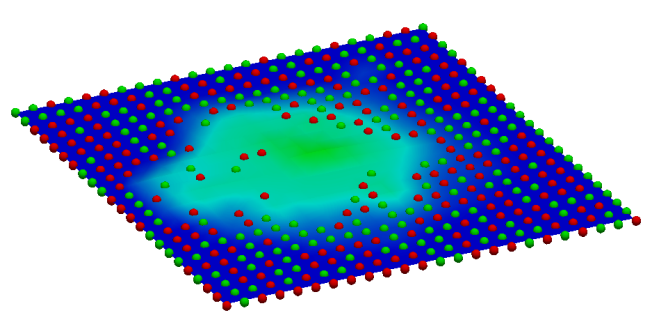

(b) Points used as stations (green), and points used as control points for calibration (red)

Figure 4: HARMONIE points used in simulation

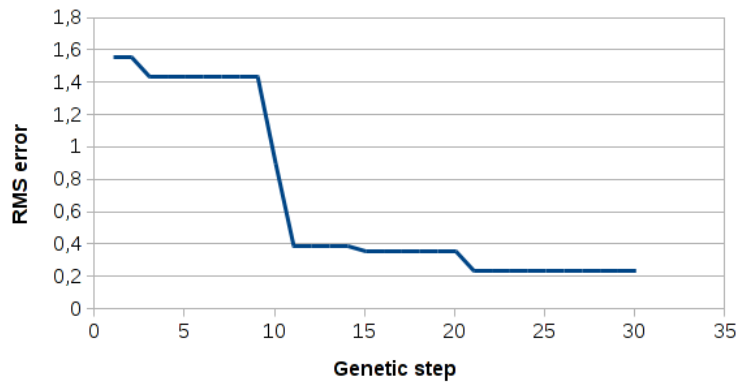

Figure 5: Error $\left(\mathrm{m} \mathrm{s}^{-1}\right)$ at each genetic step 


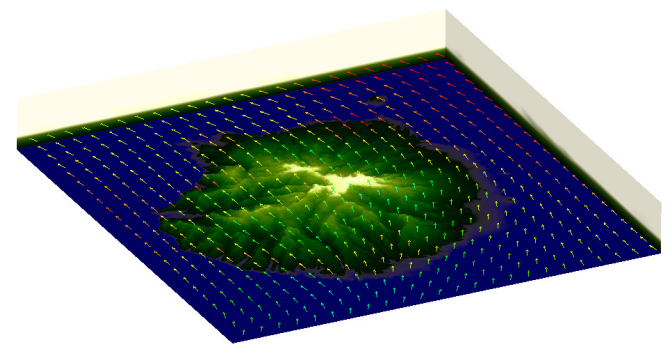

(a) HARMONIE wind field

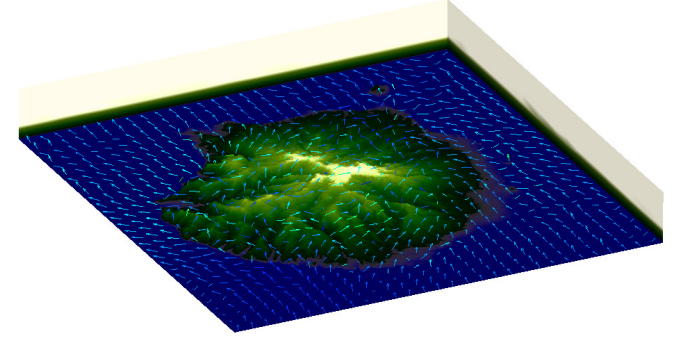

(b) Resulting wind field

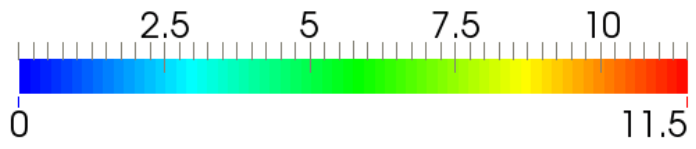

Figure 6: Wind field at $10 \mathrm{~m}\left(\mathrm{~ms}^{-1}\right)$ 


\subsection{Resulting Wind Field}

With the parameters calibrated by the genetic algorithm we can finally compute the resulting wind field.

Figure 6 shows the wind field at $10 \mathrm{~m}$ for the HARMONIE meso-scale model (a), and the resulting wind field (b). It can be observed that the HARMONIE wind field crosses the island almost ignoring the orography, while the resulting wind field changes its direction when it reaches the island.

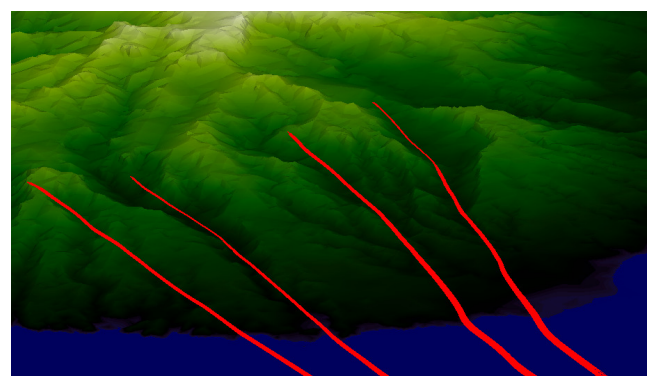

Figure 7: Streamlines of the resulting wind field

In Fig. 7 we can see a detail of the streamlines of the resulting wind field in several points. It has to be remarked that the wind field tries to align with the valleys of the island.

\subsection{Validation against Measurement Station Data}

To validate the method presented in this work, a comparison between the resulting wind field and real wind measurements has been performed.

\begin{tabular}{|c|c|c|}
\hline Station & $X$ & $Y$ \\
\hline C619X & 429982.2 & 3108577.3 \\
C629Q & 429966.6 & 3073034.7 \\
C635B & 443504.2 & 3088472.9 \\
C639X & 455377.2 & 3076514.6 \\
C639Y & 443283.4 & 3070534.1 \\
C649R & 462851.1 & 3095782.8 \\
\hline
\end{tabular}

Table 1: Measurement stations UTM coordinates

Six measurement stations have been considered. Their UTM coordinates are summarised in Table 1, and their location is represented in Fig. 8. Most stations are near the sea level, except for C635B with a height of $960 \mathrm{~m}$.

In order to conduct the comparison, a whole day forecast has been executed. The HARMONIE data used in this forecast is a $24 \mathrm{~h}$ execution starting at midnight of 2010/02/20. Measurement data are available for the whole day in all the stations except for C629Q with only 20 measurements. 


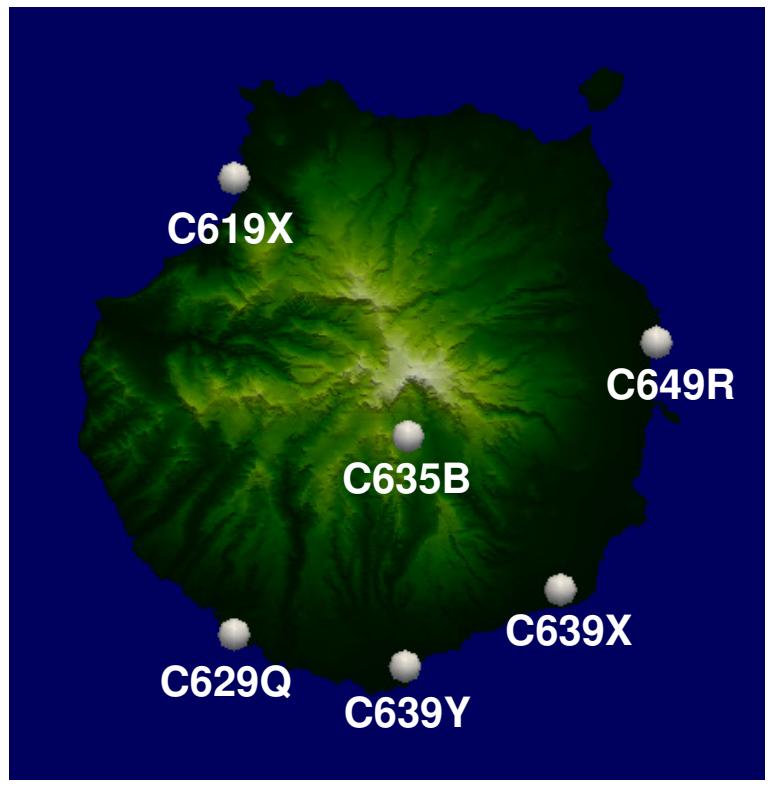

Figure 8: Location of the measurement stations

Figure 9 shows the comparisons for the stations. For each station, the instant wind velocity and the maximum wind velocity have been represented. It has to be noted that, in general, the resulting wind velocity is reasonably similar to the measured wind velocity, being in some cases closer to the maximum (for example in C629Q, C639Y); in other cases remaining within the range (C635B and C619X). The comparison at station C649R is the only one where the method has predicted, in the whole episode, a higher wind velocity than the measured one.

Examining the comparisons it can be noticed that this method smooths the wind velocity. Looking at the measured data there are abrupt changes among time steps that are not captured by the method.

A special mention has to be done of the C635B station. In the location of this station, the difference between the HARMONIE grid and the meccano mesh was greater than the tolerance, so no data from HARMONIE were used nearby. Nevertheless, the resulting wind is a good forecast, proving the feasibility of this method.

\section{Conclusions}

This paper presents a strategy to simulate the wind field forecast in complex orography locations. It is based on the coupling of the HARMONIE meso-scale model and a mass-consistent model. The results show the importance of the terrain in the resulting wind field. Genetic algorithms have proved to be useful in this kind of problems, allowing to calibrate the unknown parameter to the HARMONIE model wind field. The numerical experiment shows a reasonable behaviour of the proposed method. 


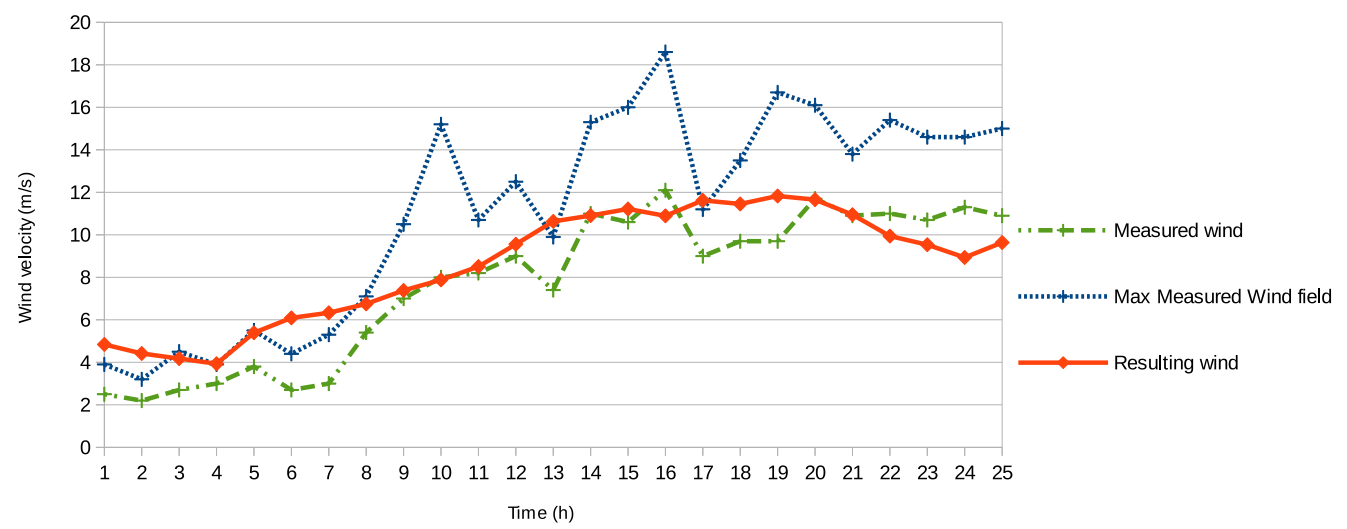

(a) C619X measurement station

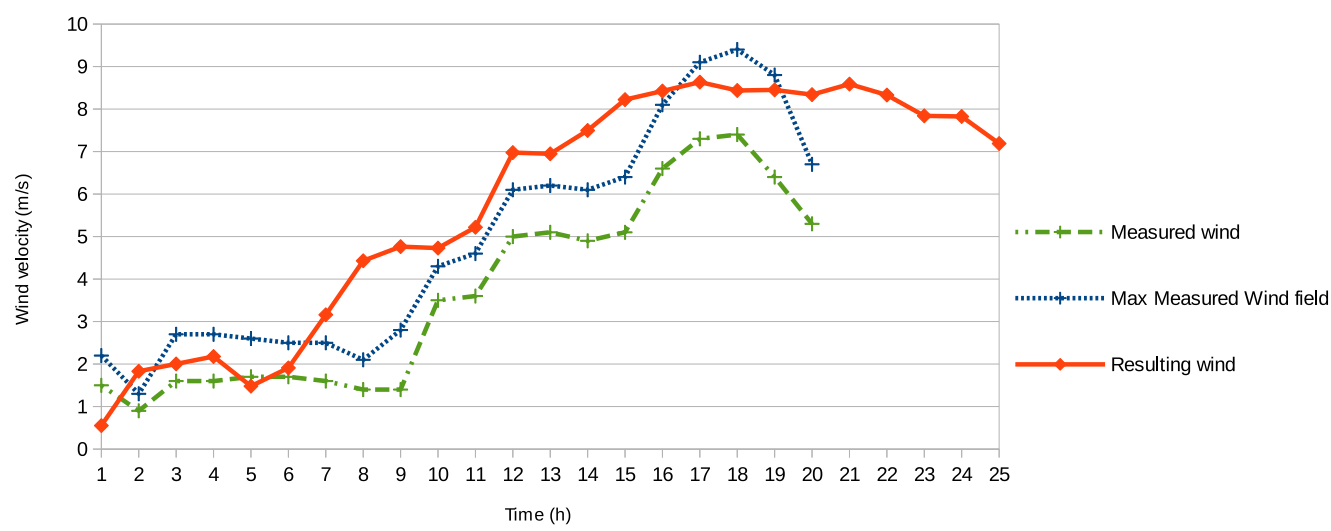

(b) C629Q measurement station

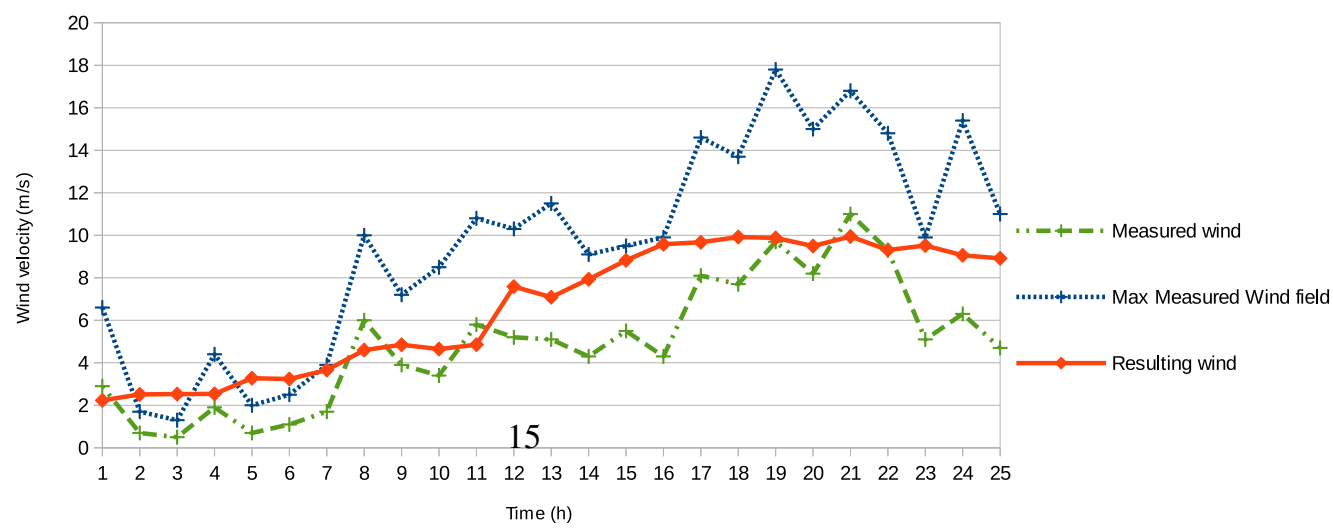

(c) C635B measurement station

Figure 9: Comparison of the wind field with the instant measured data and the maxi- 


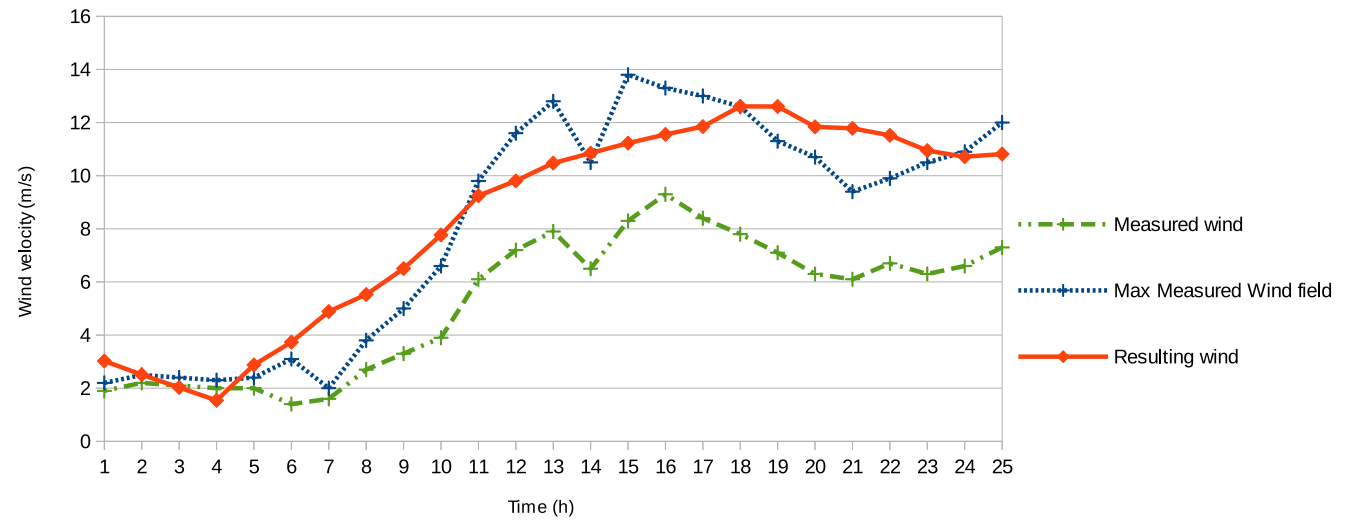

(d) C639X measurement station

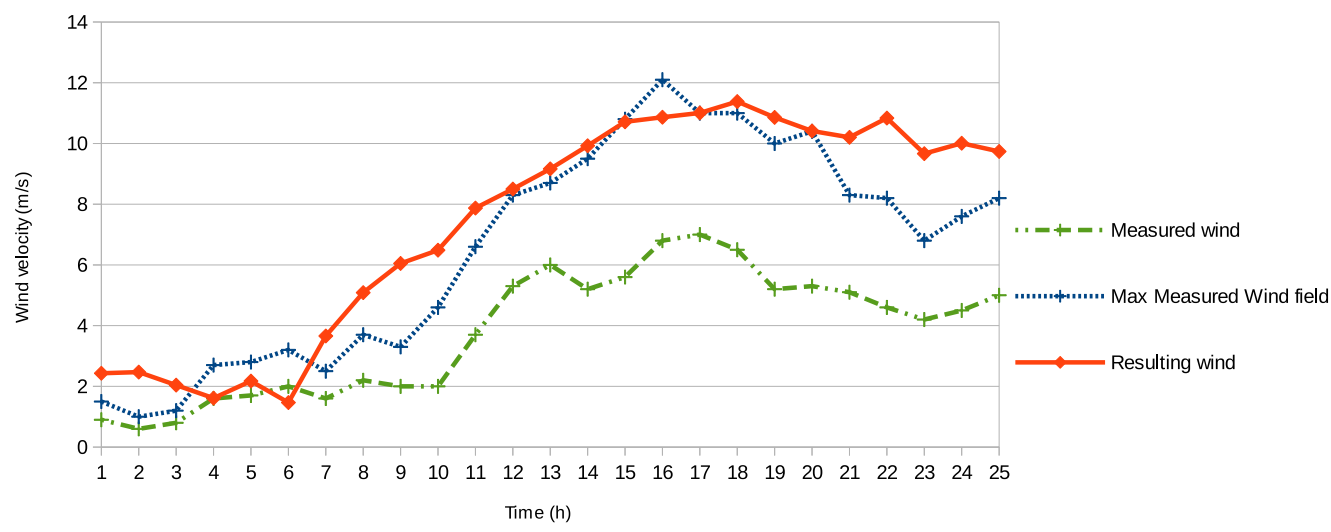

(e) C639Y measurement station

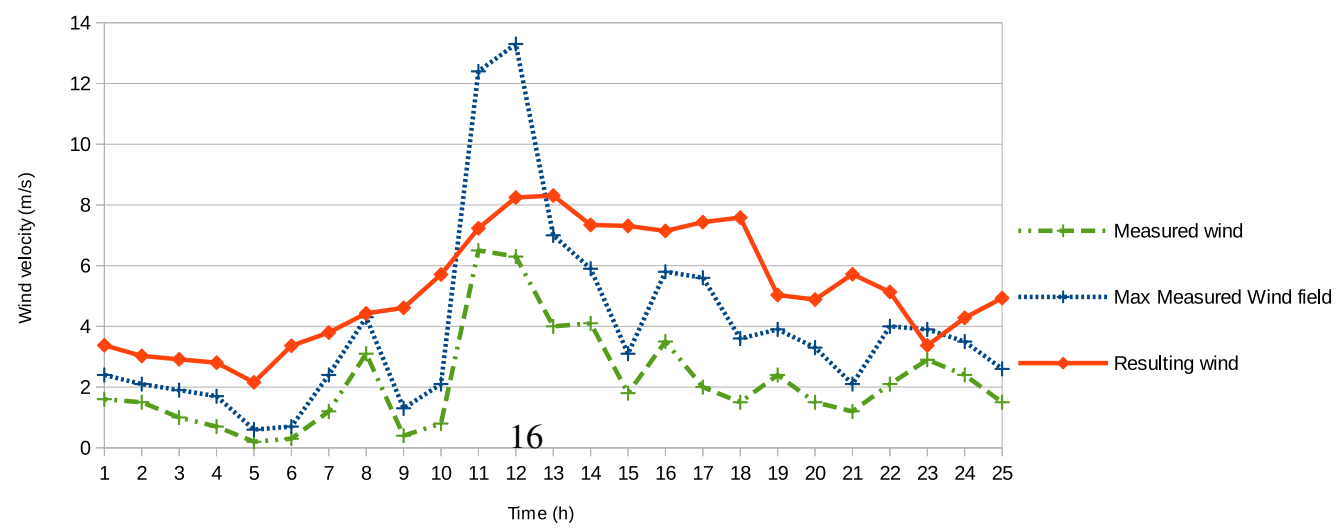

(f) C649R measurement station

Figure 9: Comparison of the wind field with the instant measured data and the maxi- 


\section{acknowledgements}

This work has been supported by the Spanish Government, "Ministerio de Ciencia e Innovación", grant contracts: CGL2011-29396-C03-01 and CGL2011-29396-C03-02, and by "Junta de Castilla León", "Consejería de Educación", Grant contract SA266A122.

\section{References}

H. Borouchaki and P. Frey. Simplification of surface mesh using hausdorff envelope. Computer Methods in Applied Mechanics and Engineering, 194(48-49):4864-4884, 2005. ISSN 0045-7825. doi: 10.1016/j.cma.2004.11.016.

R. Bubnová, G. Hello, P. Bénard, and J. Geleyn. Integration of the fully elastic equations cast in the hydrostatic pressure terrain-following coordinate in the framework of the arpege/aladin nwp system. Mon. Wea. Rev., 123:515-535, 1995. doi: 10.1175/1520-0493(1995)123<0515:IOTFEE >2.0.CO;2.

D. Carvalho, A. Rocha, C. S. Santos, and R. Pereira. Wind resource modelling in complex terrain using different mesoscaleâĂŞmicroscale coupling techniques. Applied Energy, 108(0):493-504, 2013. ISSN 0306-2619. doi: 10.1016/j.apenergy.2013.03.074.

J. M. Cascón, E. Rodríguez, J. M. Escobar, and R. Montenegro. Comparison of the meccano method with standard mesh generation techniques. Engineering with Computers, pages 1-14, 2013. ISSN 0177-0667. doi: 10.1007/s00366-013-0338-6.

L. Davis. Handbook of genetic algorithms. Van Nostrand Reinhold, New York, NY, USA, 1991. ISBN 0442001738.

J. M. Escobar, E. Rodríguez, R. Montenegro, G. Montero, and J. M. González-Yuste. Simultaneous untangling and smoothing of tetrahedral meshes. Computer Methods in Applied Mechanics and Engineering, 192(25):2775-2787, 2003. ISSN 00457825. doi: 10.1016/S0045-7825(03)00299-8.

J. M. Escobar, E. Rodríguez, R. Montenegro, G. Montero, and J. M. GonzálezYuste. SUS code: simultaneous mesh untangling and smoothing code. http://www.dca.iusiani.ulpgc.es/SUScode, 2010.

L. Ferragut, M. Asensio, and S. Monedero. A numerical method for solving convection-reaction-diffusion multivalued equations in fire spread modelling. Advances in Engineering Software, 38(6):366-371, 2007. ISSN 0965-9978. doi: 10.1016/j.advengsoft.2006.09.007.

L. Ferragut, R. Montenegro, G. Montero, E. Rodríguez, M. Asensio, and J. M. Escobar. Comparison between 2.5-D and 3-D realistic models for wind field adjustment. Journal of Wind Engineering and Industrial Aerodynamics, 98(10-11):548-558, 2010. ISSN 0167-6105. doi: 10.1016/j.jweia.2010.04.004. 
C. Fischer, T. Montmerle, L. Berre, L. Auger, and S. E. Ştefănescu. An overview of the variational assimilation in the aladin/france numerical weather-prediction system. Quarterly Journal of the Royal Meteorological Society, 131(613):3477-3492, 2005. ISSN 1477-870X. doi: 10.1256/qj.05.115.

M. S. Floater. Mean value coordinates. Computer Aided Geometric Design, 20(1): 19-27, 2003. ISSN 0167-8396. doi: 10.1016/S0167-8396(03)00002-5.

J. Garratt. Observations in the nocturnal boundary layer. Boundary-Layer Meteorology, 22(1):21-48, 1982. ISSN 0006-8314. doi: 10.1007/BF00128054.

N. Gasset, M. Landry, and Y. Gagnon. A comparison of wind flow models for wind resource assessment in wind energy applications. Energies, 5(11):4288-4322, 2012. ISSN 1996-1073. doi: 10.3390/en5114288.

J. H. Holland. Adaptation in Natural and Artificial Systems. MIT Press, Cambridge, MA, USA, 1992. ISBN 0-262-58111-6.

I. Kossaczký. A recursive approach to local mesh refinement in two and three dimensions. Journal of Computational and Applied Mathematics, 55(3):275-288, 1994. ISSN 0377-0427. doi: 10.1016/0377-0427(94)90034-5.

D. Lalas and C. Ratto. Modelling of Atmospheric Flow Fields. World Scientific Publishing, Singapore, 1996.

D. Levine. A parallel genetic algorithm for the set partitioning problem, 1994.

G. J. McRae, W. R. Goodin, and J. H. Seinfeld. Development of a second-generation mathematical model for urban air pollution-I. Model formulation. Atmospheric Environment (1967), 16(4):679-696, 1982. ISSN 0004-6981. doi: 10.1016/00046981(82)90386-9.

R. Montenegro, J. M. Cascón, J. M. Escobar, E. Rodríguez, and G. Montero. An automatic strategy for adaptive tetrahedral mesh generation. Applied Numerical Mathematics, 59(9):2203-2217, 2009. ISSN 0168-9274. doi: 10.1016/j.apnum.2008.12.010.

R. Montenegro, J. M. Cascón, E. Rodríguez, J. M. Escobar, and G. Montero. The meccano method for automatic three-dimensional triangulation and volume parametrization of complex solids. In B. Topping, J. Adam, F. Pallarés, R. Bru, and M. Romero, editors, Developments and Applications in Engineering Computational Technology, chapter 2, pages 19-48. Saxe-Coburg Publications, Stirlingshire, seventh edition, 2010. ISBN 978-1-874672-48-7. doi: 10.4203/csets.26.2.

G. Montero and N. Sanín. 3-D modelling of wind field adjustment using finite differences in a terrain conformal coordinate system. Journal of Wind Engineering and Industrial Aerodynamics, 89(5):471-488, 2001. ISSN 0167-6105. doi: 10.1016/S0167-6105(00)00075-1. 
G. Montero, R. Montenegro, and J. M. Escobar. A 3-D diagnostic model for wind field adjustment. Journal of Wind Engineering and Industrial Aerodynamics, 74-76(0): 249-261, 1998. ISSN 0167-6105. doi: 10.1016/S0167-6105(98)00022-1.

G. Montero, E. Rodríguez, R. Montenegro, J. M. Escobar, and J. M. González-Yuste. Genetic algorithms for an improved parameter estimation with local refinement of tetrahedral meshes in a wind model. Advances in Engineering Software, 36(1):3-10, 2005. ISSN 0965-9978. doi: 10.1016/j.advengsoft.2004.03.011.

B. Navascués, J. Calvo, G. Morales, C. Santos, A. Callado, A. Cansado, J. Cuxart, M. Díez, P. del Río, P. Escribà, O. García-Colombo, J. García-Moya, C. Geijo, E. Gutiérrez, M. Hortal, I. Martínez, B. Orfila, J. Parodi, E. Rodríguez, J. SánchezArriola, I. Santos-Atienza, and J. Simarro. Long-term verification of HIRLAM and ECMWF forecasts over southern europe: History and perspectives of numerical weather prediction at AEMET. Atmospheric Research, 125-126(0):20-33, 2013. ISSN 0169-8095. doi: 10.1016/j.atmosres.2013.01.010.

A. Oliver, G. Montero, R. Montenegro, E. Rodríguez, J. M. Escobar, and A. PérezFoguet. Finite element simulation of a local scale air quality model over complex terrain. Advances in Science and Research, 8:105-113, 2012. doi: 10.5194/asr-8105-2012.

A. Oliver, G. Montero, R. Montenegro, E. Rodríguez, J. M. Escobar, and A. PérezFoguet. Adaptive finite element simulation of stack pollutant emissions over complex terrains. Energy, 49(0):47-60, 2013. ISSN 0360-5442. doi: 10.1016/j.energy.2012.10.051.

H. Panofsky and J. Dutton. Atmospheric turbulence. Models and methods for engineering applications. Wiley, New York, 1984.

E. Rodríguez. Modelización numérica de campos de viento mediante elementos finitos en 3-D. PhD thesis, Instituto Universitario de Sistemas Inteligentes y Aplicaiones Numéricas en Ingenierı (IUSIANI), Universidad de Las Palmas de Gran Canaria (ULPGC), 2004.

E. Rodríguez, G. Montero, J. M. Escobar, R. Montenegro, and A. Oliver. Wind3D. http://www.dca.iusiani.ulpgc.es/Wind3D, 2012.

Y. Seity, P. Brousseau, S. Malardel, G. Hello, P. Bénard, F. Bouttier, C. Lac, and V. Masson. The arome-france convective-scale operational model. Mon. Wea. Rev., 139: 976-991, 2011. doi: 10.1175/2010MWR3425.1.

W. Spears. On the virtues of parameterized uniform crossover, 1991.

S. Wan, Z. Yin, K. Zhang, H. Zhang, and X. Li. A topology-preserving optimization algorithm for polycube mapping. Computers \& Graphics, 35(3):639-649, 2011. ISSN 0097-8493. doi: 10.1016/j.cag.2011.03.018. 
D. Whitley. The genitor algorithm and selection pressure: Why rank-based allocation of reproductive trials is best. In Proceedings of the Third International Conference on Genetic Algorithms, pages 116-121. Morgan Kaufmann, 1989.

P. Zannetti. Air Pollution Modeling. Computational Mechanics Publications, Boston, 1990. 